\title{
Validação da Versão Portuguesa do Questionário EHP-30 (The Endometriosis Health Profile-30)
}

\author{
Validation of the Portuguese Version of EHP-30 (The Endometriosis Health \\ Profile-30)
}

\author{
Cristina NOGUEIRA-SILVA $\triangle^{1,2,3}$, Patrício $\operatorname{COSTA}^{1,2}$, Carla MARTINS ${ }^{4}$, Sónia BARATA ${ }^{5}$, Conceição ALHO ${ }^{5}$, Carlos \\ CALHAZ-JORGE ${ }^{5,6}$, Filipa OSÓRIO 5,6 \\ Acta Med Port 2015 May-Jun;28(3):347-356
}

\section{RESUMO}

Introdução: O Endometriosis Health Profile Questionnaire-30 é atualmente o questionário mais utilizado para avaliação da qualidade de vida em mulheres com endometriose. O objetivo do presente estudo é avaliar as propriedades psicométricas e validar a versão portuguesa do Endometriosis Health Profile Questionnaire-30.

Material e Métodos: Amostra sequencial de conveniência, constituída por 152 doentes com endometriose, de um centro de referência no país, que autopreencheram um questionário sociodemográfico, a versão portuguesa do Endometriosis Health Profile Questionnaire-30 e do Short Form Health Survey 36 Item-versão 2. Procedeu-se a análise estatística apropriada, com estatística descritiva, análise fatorial, avaliação da consistência interna, correlação item-total e validade convergente (usando o Short Form Health Survey 36 Item-versão 2).

Resultados: A análise fatorial confirmou a validade da estrutura em cinco dimensões do questionário central, explicando uma variância total de $83,2 \%$. A correlação item-total apresentou resultados aceitáveis em todos os itens e a consistência interna foi elevada, com a Cronbach variando de 0,876 a 0,981 nas dimensões do questionário central, e de 0,863 a 0,951 no modular. Demonstrou-se associação negativa significativa entre as dimensões similares do Endometriosis Health Profile Questionnaire-30 e do Short Form Health Survey 36 Item-versão 2. A taxa de preenchimento do questionário foi elevada para todas as dimensões. A perda do bem-estar emocional (no questionário central) e a infertilidade (no modular) apresentaram as pontuações médias mais elevadas e, consequentemente, impacto mais negativo sobre a qualidade de vida.

Discussão: São necessários estudos para avaliar a fiabilidade teste-reteste e a sensibilidade à mudança desta versão portuguesa do Endometriosis Health Profile Questionnaire-30.

Conclusão: Este estudo demonstra que a versão portuguesa do Endometriosis Health Profile Questionnaire-30 é um instrumento adaptado, validado e bem aceite para a avaliação da qualidade de vida das mulheres portuguesas com endometriose.

Palavras-chave: Endometriose; Qualidade de Vida; Questionários; Portugal.

\section{ABSTRACT}

Introduction: Endometriosis Health Profile Questionnaire-30 is currently the most used questionnaire for quality of life measurement in women with endometriosis. The aim of this study is to evaluate the psychometric properties and to validate the Portuguese Endometriosis Health Profile Questionnaire-30 version.

Material and Methods: A sequential sample of 152 patients with endometriosis, followed in a Portugal reference center, were asked to complete a questionnaire on social and demographic features, the Portuguese version of the Endometriosis Health Profile Questionnaire-30 and of the Short Form Health Survey 36 Item - version 2. Appropriate statistical analysis was performed using descriptive statistics, factor analysis, internal consistency, item-total correlation and convergent validity.

Results: Factorial analysis confirmed the validity of the five-dimension structure of the Endometriosis Health Profile Questionnaire-30 core questionnaire, which explained $83.2 \%$ of the total variance. All item-total correlations presented acceptable results and high internal consistency, with Cronbach's alpha ranging between 0.876 and 0.981 for the core questionnaire and between 0.863 and 0.951 for the modular questionnaire. Significant negative associations between similar scales of Endometriosis Health Profile Questionnaire-30 and Short Form Health Survey 36 Item - version 2 were demonstrated. Data completeness achieved was high for all dimensions. The emotional well-being scale in the core questionnaire and the infertility scale in the modular section had the highest median scores, and therefore the most negative impact on the quality of life of participating women.

Discussion: The test-retest reliability and responsiveness of the questionnaire should be evaluated in future studies.

Conclusion: The present study demonstrates that the Portuguese version of the Endometriosis Health Profile Questionnaire-30 is a valid, reliable and acceptable tool for evaluating the health-related quality of life of Portuguese women with endometriosis.

Keywords: Endometriosis; Quality of Life; Questionnaires; Portugal.

\footnotetext{
1. Instituto de Investigação em Ciências da Vida e da Saúde. Escola de Ciências da Saúde. Universidade do Minho. Braga. Portugal.

2. Instituto de Investigação em Ciências da Vida e da Saúde/3B's - Laboratório Associado do Governo Português. Braga/ Guimarães. Portugal.

3. Serviço de Ginecologia e Obstetrícia. Hospital de Braga. Braga. Portugal.

4. AlcaisFisio. Lisboa. Portugal.

5. Clínica Universitária de Obstetrícia e Ginecologia. Centro Hospitalar Lisboa Norte - Hospital Universitário de Santa Maria. Lisboa. Portugal

6. Departamento de Obstetrícia e Ginecologia. Faculdade de Medicina. Universidade de Lisboa. Lisboa. Portugal.

$\square$ Autor correspondente: Cristina Nogueira-Silva. cristinasilva@ecsaude.uminho.pt

Recebido: 31 de Agosto de 2014 - Aceite: 11 de Maio de 2015 | Copyright @ Ordem dos Médicos 2015
} 


\section{INTRODUÇÃO}

A endometriose é uma doença crónica, frequentemente progressiva e recidivante, caraterizada pela presença de tecido glandular e estroma endometrial fora do útero. Embora a sua prevalência seja difícil de determinar, estimase que afete cerca de $10 \%$ das mulheres em idade reprodutiva, o que corresponde a 176 milhões de mulheres com endometriose em todo o mundo. ${ }^{1}$ Clinicamente pode manifestar-se por dismenorreia, dor pélvica crónica, dispareunia, disúria, disquésia, infertilidade e hemorragia uterina anormal, podendo condicionar importante morbilidade física, emocional e social e, consequentemente, diminuição da qualidade de vida. ${ }^{2-5}$

A qualidade de vida relacionada com a saúde é um conceito multidimensional e dinâmico, que engloba aspetos físicos, psicológicos e sociais relacionados com uma doença ou o seu tratamento., ${ }^{3,6}$ Dado que a endometriose afeta globalmente a vida da mulher, a avaliação da eficácia de um determinado tratamento baseada apenas em meIhoria da dor e das taxas de fertilidade, apresenta-se como insuficiente..$^{7,8}$ Para além disso, é amplamente reconhecida a falta de correlação entre a intensidade dos sintomas e a gravidade e extensão das lesões. ${ }^{9}$ Assim, a avaliação subjetiva pela doente da sua qualidade de vida é fulcral na abordagem da endometriose.

Recentemente, vários estudos têm demonstrado uma redução da qualidade de vida em mulheres com endometriose. ${ }^{10,11}$ Contudo, esses estudos utilizaram instrumentos genéricos (nomeadamente Nottingham Health Profile, Short Form-36, Short Form-12), que demonstraram não ser suficientemente sensíveis para detetar alterações e respostas ao tratamento em doenças específicas e complexas, como a endometriose., ${ }^{4,12-15}$ Assim, surgiu a necessidade de desenvolvimento de instrumentos específicos para a endometriose, que sejam sensíveis a alterações da qualidade de vida e do estado de saúde das pacientes com esta doença. ${ }^{2}$

O Endometriosis Health Profile Questionnaire-30 (EHP30) é atualmente o questionário mais utilizado e validado para avaliação da qualidade de vida em mulheres com endometriose, ${ }^{16,17}$ sendo recomendado quer pela Sociedade Americana de Medicina da Reprodução (ASMR), quer pela Sociedade Europeia para a Reprodução Humana e Embriologia para a investigação em qualidade de vida relacionada com a saúde na endometriose. ${ }^{18}$ De facto, foi já demonstrado que o EHP-30 é sensível a alterações no estado de saúde em doentes com endometriose ao longo do tempo. 19,20

O EHP-30 foi desenvolvido em 2001 no Reino Unido, através de entrevistas exploratórias a doentes com diagnóstico cirúrgico de endometriose. ${ }^{2} \mathrm{O}$ questionário, de autopreenchimento, é composto por uma parte central, com 30 itens, que avaliam cinco dimensões (dor, controlo e impotência, bem-estar emocional, suporte social e autoimagem), e por um questionário modular, com 23 itens distribuídos por 6 dimensões, que podem não se aplicar a todas as mulheres, e como tal o seu preenchimento é opcional (vida no trabalho, relação com os filhos, relações sexuais, rela- ção com os médicos, tratamento e infertilidade). ${ }^{2,21}$ Cada escala é transformada num sistema de pontuação que vai de 0 a 100, sendo que quanto menor a pontuação, melhor a qualidade de vida da mulher. O EHP-30 encontra-se já validado no contexto americano e nas versões australiana, holandesa, italiana, chinesa, iraniana e portuguesa do Brasil. 8,16,17,21-24 Recentemente, o processo de tradução de uma versão do EHP-30 culturalmente adaptada a Portugal foi desenvolvido. ${ }^{25,26}$ Contudo, até à data esse instrumento não foi validado. Tal, permitiria avaliar a qualidade de vida das doentes portuguesas, bem como a sua comparação com diferentes estudos internacionais, em que o mesmo instrumento fosse utilizado.

Assim, o objetivo do presente estudo foi avaliar as propriedades psicométricas e proceder à validação da versão portuguesa do EHP-30, em mulheres portuguesas com diagnóstico de endometriose, num centro de referência do país.

\section{MATERIAL E MÉTODOS \\ Adaptação do questionário}

O processo de tradução (equivalência semântica e de conteúdo obtida por duas traduções independentes e duas retroversões, obtenção de uma versão de consenso e revisão por clínicos) e avaliação da validade de conteúdo (painel de 20 mulheres com endometriose) foi previamente realizado. ${ }^{25,26}$ No trabalho atual foi utilizada essa versão traduzida e culturalmente adaptada, após solicitação de autorização aos seus autores.

\section{Estudos psicométricos e validação}

O estudo foi aprovado pela Comissão de Ética do Centro Hospitalar Lisboa Norte (CHLN) - Hospital Universitário de Santa Maria.

Todas as doentes incluídas no estudo foram informadas do seu objetivo, do seu carácter anónimo e confidencial, e de que a sua participação seria voluntária, podendo recusar ou a qualquer momento interromper a participação no estudo, sem nenhum tipo de penalização ou prejuízo por tal facto, nomeadamente no que diz respeito aos seus direitos assistenciais. Após devidamente esclarecidas, todas as participantes assinaram o consentimento informado antes da aplicação dos questionários.

\section{Amostra e questionários}

Amostra sequencial de conveniência, constituída por 152 doentes com endometriose, em idade reprodutiva e com idade superior a 18 anos, seguidas na consulta de Ginecologia Cirúrgica do Departamento/ Clínica Universitária de Obstetrícia e Ginecologia do CHLN/ Hospital Universitário de Santa Maria. Esta é uma consulta de referência de doentes com endometriose, sendo por isso frequentada por doentes residentes em todo o país (embora com predomínio para a região de Lisboa e Vale do Tejo).

O tamanho da amostra foi determinado considerando as recomendações de Hair et al. ${ }^{27}$ Isto é, dimensão amos- 
tral superior a 100 participantes e cinco vezes mais observações do que o número de itens a serem analisados. Foi considerado critério de exclusão a presença de outra doença física ou mental, com implicação importante na qualidade de vida.

Foram aplicados a todas as doentes os seguintes questionários: questionário sociodemográfico (idade, nacionalidade, etnia, escolaridade, profissão e estado civil), versão portuguesa do EHP-30 e versão portuguesa do Short Form Health Survey 36 Item - versão 2 (SF-36v2). O SF-36v2 foi já validado em português e é constituído por 36 itens, englobados em oito dimensões (funcionamento físico, desempenho físico, dor corporal, saúde em geral, vitalidade, funcionamento social, desempenho emocional e saúde mental), que permitem avaliar aspetos da saúde física e mental. Cada dimensão é traduzida numa pontuação que vai de 0 a 100, sendo que quanto maior a pontuação, meIhor a qualidade de vida da mulher. ${ }^{28}$

Os questionários foram auto-preenchidos pelas doentes em tratamento (expectante, médico ou cirúrgico) da endometriose, na sala de espera da consulta, entre 8 de abril de 2013 e 31 de março de 2014, aquando da sua presença na consulta por motivos clínicos.

Foram, também, recolhidos dados clínicos, através do preenchimento de um formulário clínico pelo médico responsável pela doente, incluindo sintomatologia apresentada nas quatro semanas prévias à consulta e estádio da endometriose (de acordo com a classificação da ASRM). ${ }^{29}$

\section{Análise estatística}

Foram utilizados seis critérios para avaliação das propriedades psicométricas do questionário, nomeadamente análise fatorial exploratória, avaliação da consistência interna, correlação item-total, validade convergente, taxa de preenchimento e distribuição das pontuações. Para tal, os dados foram introduzidos e analisados no Statistical Package for Social Sciences (SPSS ${ }^{\circledR}$, versão 22.0). Procedeu-se previamente a uma análise descritiva, com recurso à distribuição de frequência, às medidas de tendência central e às medidas de dispersão, tendo em conta as variáveis em estudo. Os resultados referentes a variáveis quantitativas são apresentados como média \pm desvio-padrão. Para verificar a estrutura fatorial do questionário, foi realizada análise fatorial exploratória do questionário central do EHP-30, em componentes principais com rotação Varimax. Para avaliação da consistência interna para cada escala do questionário central e modular foi utilizado o coeficiente alfa de Cronbach, considerando-se valores $\geq 0,7$ aceitáveis. ${ }^{27}$ Foram considerados como aceitáveis valores de correlação item-total acima de 0,4. ${ }^{27}$ Para avaliar a validade convergente, à semelhança do estudo que descreveu o desenvolvimento da versão original, bem como versões de outras línguas, foi hipotetizada uma correlação significativa entre as dimensões do EHP-30 e do SF-36v2.2,5,23 A validade convergente foi verificada pelo coeficiente de correlação de Pearson. Admitiu-se significância estatística para valores de $p<0,05$.

\section{RESULTADOS}

A idade média das 152 mulheres com endometriose que constituem a amostra é $34,7 \pm 6,1$ anos (mínimo 19; máximo 49). São maioritariamente de nacionalidade portuguesa $(96,1 \%)$, etnia caucasiana $(88,2 \%)$ e vivem em união conjugal (casadas ou união de facto, $69,6 \%$ ). Relativamente às habilitações literárias, 40,8\% $(n=62)$ concluíram o ensino secundário e $38,2 \%(n=58)$ têm habilitações iguais ou superiores a uma licenciatura. De realçar que $78,9 \%$ (n = 120) são profissionalmente ativas (Tabela 1).

Relativamente a fatores clínicos, salienta-se que $66,4 \%$ ( $n=101$ ) das mulheres da amostra apresentavam endometriose no estádio IV, de acordo com a classificação da ASMR. Relativamente à sintomatologia apresentada nas últimas quatro semanas (em relação ao momento de preenchimento do questionário), a maioria das mulheres referia dismenorreia $(63,6 \%)$, dispareunia $(54,8 \%)$, dor pélvica crónica $(50,0 \%)$, astenia $(59,2 \%)$ e alterações gastrointestinais $(54,6 \%)$ (Tabela 1$)$.

\section{Análise fatorial exploratória}

Foi realizada a análise fatorial do questionário central especificando um máximo de cinco fatores, a fim de determinar se a estrutura atual refletia a estrutura da versão original do EHP-30. O trabalho original que documentou o desenvolvimento do EHP-30 assumiu que não existia nenhuma correlação entre os fatores e, consequentemente utilizou a rotação Varimax. ${ }^{2}$ Assim, a rotação Varimax foi também utilizada neste trabalho. Os itens com uma saturação fatorial mínima de 0,4 na análise de componentes principais foram mantidos para a análise fatorial (ou seja, todos os itens foram mantidos) (Tabela 2).

A análise integrando cinco fatores (todos com valores próprios superiores a 1) permitiu obter uma variância de $83,2 \%$. A percentagem de variância explicada por cada fator foi $36,8 \%$ para a dor, $18,6 \%$ para controlo e impotência, $12,2 \%$ para bem-estar emocional, $8,2 \%$ para suporte social e 7,4\% para autoimagem. Todos os itens saturaram de forma mais saliente na dimensão correta, com a exceção de seis (Tabela 2): o item 'Em geral, sentiu-se mal' associou-se à dimensão dor; os itens 'Sintomas não estão a melhorar', 'Incapacidade de controlar sintomas', 'Sintomas a controlar a vida' e 'Sintomas a tirar a vida' associaram-se à dimensão dor para além da dimensão hipotetizada (controlo e impotência); por fim, o item 'Incapacidade de dizer como se sente' associou-se à dimensão hipotetizada (suporte social) para além da dimensão bem-estar emocional.

Após encontrarmos as cinco dimensões de primeira ordem submetemo-las a nova análise fatorial, com o objetivo de verificar se emergia uma componente única de segunda ordem. Os resultados indicam-nos que é possível gerar um único componente, que explica $78,0 \%$ de variância. Tal indica que as dimensões podem ser somadas para criar uma pontuação única. A saturação fatorial dos componentes principais para as cinco dimensões foi elevada (dor: 0,877 ; controlo e impotência: 0,906; bem-estar emocional: 0,903; suporte social: 0,897; autoimagem: 0,831). 
Tabela 1 - Caraterísticas sócio-demográficas e clínicas das doentes com endometriose $(n=152)$

\begin{tabular}{|c|c|}
\hline \multicolumn{2}{|l|}{ Caraterísticas Sócio-Demográficas } \\
\hline Idade* & $34,7 \pm 6,1 \quad(19-49)$ \\
\hline \multicolumn{2}{|l|}{ Nacionalidade, n (\%) } \\
\hline Portuguesa & $146(96,1 \%)$ \\
\hline Outras $^{a}$ & $6(3,9 \%)$ \\
\hline \multicolumn{2}{|l|}{ Etnia, n (\%) } \\
\hline Caucasiana & $134(88,2 \%)$ \\
\hline Negra & $12(7,9 \%)$ \\
\hline Outras & $6(3,9 \%)$ \\
\hline \multicolumn{2}{|l|}{ Escolaridade, n (\%) } \\
\hline $1^{\circ}$ Ciclo & $0(0,0 \%)$ \\
\hline $2^{\circ}$ Ciclo & $2(1,3 \%)$ \\
\hline $3^{\circ}$ Ciclo & $20(13,2 \%)$ \\
\hline Ensino Secundário & $62(40,8 \%)$ \\
\hline Bacharelato & $10(6,6 \%)$ \\
\hline Licenciatura/ Mestrado Integrado & $48(31,6 \%)$ \\
\hline Mestrado/ Doutoramento & $10(6,6 \%)$ \\
\hline \multicolumn{2}{|l|}{ Profissão, n (\%) } \\
\hline Ativa & $120(78,9 \%)$ \\
\hline Estudante & $6(3,9 \%)$ \\
\hline Inativa ${ }^{b}$ & $26(17,1 \%)$ \\
\hline \multicolumn{2}{|l|}{ Estado civil, n (\%) } \\
\hline Solteira & $33(21,7 \%)$ \\
\hline Casada/ União de facto & $106(69,6 \%)$ \\
\hline Divorciada/ Separada & $12(7,9 \%)$ \\
\hline Viúva & $1(0,7 \%)$ \\
\hline
\end{tabular}

\begin{tabular}{lc}
\hline Caraterísticas Clínicas & \\
\hline Classificação Endometriose, $\mathbf{n}(\%)^{c}$ & \\
\hline Estádio I & $1(0,7 \%)$ \\
Estádio II & $13(8,6 \%)$ \\
Estádio III & $37(24,3 \%)$ \\
Estádio IV & $101(66,4 \%)$
\end{tabular}

Sintomatologia, $\mathbf{n}(\%)^{\mathrm{d}}$

Dismenorreia $(n=88)$

$56(63,6 \%)$

Disúria

$23(15,1 \%)$

Disquésia

$57(37,5 \%)$

Dispareunia $(n=115)$

$63(54,8 \%)$

Dor pélvica crónica

$76(50,0 \%)$

Astenia

$90(59,2 \%)$

Alterações gastrointestinais

$83(54,6 \%)$

Hemorragia uterina anormal

$37(24,3 \%)$

Tempo desde primeiros sintomas*

$9,6 \pm 7,4(1-31)$

"média \pm desvio-padrão (valor mínimo e máximo); Idade, tempo desde primeiros sintomas: anos; a: de outra nacionalidade mas a residir em Portugal há mais de 5 anos; inclui desempregadas e domésticas; c: Classificação de acordo com a American Society of Reproductive Medicine, estádio I - mínima, estádio II - ligeira, estádio III - moderada, estádio IV - grave ${ }^{31}$; ${ }^{\text {d: }} \mathrm{n}$ diferente para dismenorreia e dispareunia, dado em determinadas situações não se aplicar.
Correlação item-total e avaliação da consistência interna

A correlação item-total foi superior a 0,4 em todos os itens do questionário central (variação: 0,656 - 0,951; Tabela 3) e modular (variação: 0,573 - 0,937; Tabela 4) do EHP30 , o que é indicativa de uma boa consistência item-total.

$\mathrm{Na}$ avaliação da consistência interna, o alfa de Cronbach foi superior a 0,7 para todas as dimensões, variando entre 0,876 e 0,981 nas dimensões do questionário central (Tabela 3) e entre 0,863 e 0,951 nas dimensões do questionário modular (Tabela 4).

\section{Validade convergente}

Quando foi aplicada a correlação de Pearson entre dimensões do EHP-30 e do SF-36v2, encontrou-se uma correlação significativa para todas as dimensões avaliadas, que foi máxima entre a dimensão dor do EHP-30 e a dimensão dor corporal do SF-36v2 (Pearson $r=-0,739$ ); (Tabela 5).

Taxa de preenchimento do questionário, estatística descritiva e sensibilidade das diferentes dimensões

O tempo médio de preenchimento do EHP-30 foi 13,84 $\pm 6,1$ minutos (mínimo três minutos; máximo 30 minutos).

A taxa de preenchimento pelas 152 mulheres da amostra de todas as perguntas do questionário central foi elevada, variando de $96,7 \%$ na dimensão dor a $100 \%$ nas dimensões suporte social e autoimagem. No que diz respeito ao questionário modular, o $\mathrm{n}$ passível de responder a cada dimensão é dependente desta, dado que nem todas as dimensões se aplicam a todas as doentes (por exemplo: relação com os filhos não se aplica às mulheres que não têm filhos). Assim, de acordo com o n possível, a taxa de preenchimento variou entre $93,1 \%$ na dimensão relação com os filhos e $98,6 \%$ na dimensão infertilidade.

A Tabela 6 apresenta estatística descritiva e os valores de assimetria e curtose para as dimensões que constituem a escala EHP-30. À exceção da relação com os médicos, todas as dimensões apresentam valores de achatamento negativos (distribuição platicúrtica) e valores de assimetria próximos de zero. Desta forma podemos constatar que, à exceção da relação dos doentes com os médicos, as dimensões não apresentam problemas de sensibilidade ou normalidade relevantes. Relativamente ao questionário central, a dimensão bem-estar emocional apresentou a maior pontuação média $(41,2)$ e portanto o impacto mais negativo sobre a qualidade de vida. Por sua vez, a dimensão autoimagem apresentou a menor pontuação média $(34,1)$. No questionário modular, a dimensão infertilidade apresentou a maior pontuação média $(55,9)$, indicando maior repercussão negativa sobre a qualidade de vida, e a relação com os médicos a menor $(12,8)$ (Tabela 6$)$.

\section{DISCUSSÃO}

No presente estudo procedeu-se à avaliação psicométrica da versão portuguesa do EHP-30, que tinha sido apenas previamente traduzida, ${ }^{25,26}$ de acordo com as recomendações da International Society for Pharmacoeconomics and Outcomes Research. ${ }^{30}$ Tal, permite a validação do EHP-30 
Tabela 2 - Análise fatorial do questionário central do EHP-30 (análise de componentes principais com rotação Varimax)

\begin{tabular}{|c|c|c|c|c|c|c|}
\hline & \multirow[t]{2}{*}{ Comunalidades } & \multicolumn{5}{|c|}{ Saturação Fatorial } \\
\hline & & Dor & $\begin{array}{l}\text { Controlo e } \\
\text { impotência }\end{array}$ & $\begin{array}{l}\text { Bem-estar } \\
\text { emocional }\end{array}$ & $\begin{array}{l}\text { Suporte } \\
\text { social }\end{array}$ & Autoimagem \\
\hline 1. Incapacidade de ir a acontecimentos sociais & 0,844 & 0,808 & & & & \\
\hline 2. Incapacidade de fazer tarefas domésticas & 0,861 & 0,843 & & & & \\
\hline 3. Dificuldades em estar em pé & 0,874 & 0,868 & & & & \\
\hline 4. Dificuldades em sentar-se & 0,794 & 0,831 & & & & \\
\hline 5. Dificuldades em andar & 0,892 & 0,859 & & & & \\
\hline 6. Dificuldades em fazer exercício ou atividades de lazer & 0,883 & 0,854 & & & & \\
\hline 7. Perda de apetite e/ou incapaz de comer & 0,781 & 0,766 & & & & \\
\hline 8. Incapacidade de dormir bem & 0,842 & 0,816 & & & & \\
\hline 9. Necessidade de ir para a cama/ deitar-se & 0,830 & 0,815 & & & & \\
\hline 10. Incapacidade de fazer as coisas que queria & 0,923 & 0,860 & & & & \\
\hline 11. Incapacidade de lidar com as dores & 0,859 & 0,759 & & & & \\
\hline 12. Em geral, sentiu-se mal & 0,841 & 0,801 & & & & \\
\hline 13. Sintomas não estão a melhorar & 0,857 & 0,624 & 0,508 & & & \\
\hline 14. Incapacidade de controlar sintomas & 0,874 & 0,649 & 0,496 & & & \\
\hline 15. Incapacidade de esquecer os sintomas & 0,754 & & 0,726 & & & \\
\hline 16. Sintomas a controlar a vida & 0,879 & 0,623 & 0,520 & & & \\
\hline 17. Sintomas a tirar a vida & 0,872 & 0,550 & 0,479 & & & 0,458 \\
\hline 18. Sentir-se deprimida & 0,834 & & & 0,729 & & \\
\hline 19. Sentir-se com vontade de chorar & 0,854 & & & 0,701 & & \\
\hline 20. Sentir-se extremamente infeliz & 0,770 & & & 0,661 & & 0,404 \\
\hline 21. Alterações de humor & 0,877 & & & 0,839 & & \\
\hline 22. Sentir-se mal-humorada ou irritável & 0,870 & & & 0,811 & & \\
\hline 23. Sentir-se violenta ou agressiva & 0,658 & & & 0,635 & & \\
\hline 24. Incapacidade de dizer como se sente & 0,747 & & & 0,515 & 0,425 & \\
\hline 25. Sentir que os outros não compreendem & 0,875 & & & 0,448 & 0,674 & \\
\hline 26. Sentir que os outros pensam que se está a lamuriar & 0,889 & & & & 0,693 & \\
\hline 27. Sentir-se sozinha & 0,738 & & & & 0,548 & 0,499 \\
\hline 28. Não poder vestir as roupas que queria & 0,778 & & & & & 0,785 \\
\hline 29. Sentir aparência afetada & 0,821 & & & & & 0,772 \\
\hline 30. Falta de confiança & 0,775 & & & & & 0,671 \\
\hline$\alpha$-Cronbach & & 0,878 & 0,908 & 0,902 & 0,901 & 0,839 \\
\hline Valores próprios & & 11,05 & 5,57 & 3,65 & 2,45 & 2,22 \\
\hline $\begin{array}{l}\text { Variância total explicada por cada fator } \\
\text { (acumulada) }\end{array}$ & & $36,8 \%$ & $\begin{array}{c}18,6 \% \\
(55,4 \%)\end{array}$ & $\begin{array}{c}12,2 \% \\
(67,6 \%)\end{array}$ & $\begin{array}{c}8,2 \% \\
(75,8 \%)\end{array}$ & $\begin{array}{c}7,4 \% \\
(83,2 \%)\end{array}$ \\
\hline
\end{tabular}


Tabela 3 - Avaliação da consistência interna do questionário central do EHP-30 ( $\alpha$ - Cronbach).

\begin{tabular}{|c|c|}
\hline Dimensões & Correlação item-total \\
\hline \multicolumn{2}{|l|}{ Dor $(\alpha=0,981 ; n=147)$} \\
\hline 1. Incapacidade de ir a acontecimentos sociais & 0,901 \\
\hline 2. Incapacidade de fazer tarefas domésticas & 0,913 \\
\hline 3. Dificuldades em estar em pé & 0,919 \\
\hline 4. Dificuldades em sentar-se & 0,853 \\
\hline 5. Dificuldades em andar & 0,923 \\
\hline 6. Dificuldades em fazer exercício ou atividades de lazer & 0,918 \\
\hline 7. Perda de apetite e/ou incapaz de comer & 0,848 \\
\hline 8. Incapacidade de dormir bem & 0,898 \\
\hline 9. Necessidade de ir para a cama/ deitar-se & 0,889 \\
\hline 10. Incapacidade de fazer as coisas que queria & 0,951 \\
\hline 11. Incapacidade de lidar com as dores & 0,892 \\
\hline \multicolumn{2}{|l|}{ Controlo e impotência $(\alpha=0,947 ; n=150)$} \\
\hline 12. Em geral, sentiu-se mal & 0,802 \\
\hline 13. Sintomas não estão a melhorar & 0,895 \\
\hline 14. Incapacidade de controlar sintomas & 0,912 \\
\hline 15. Incapacidade de esquecer os sintomas & 0,656 \\
\hline 16. Sintomas a controlar a vida & 0,913 \\
\hline 17. Sintomas a tirar a vida & 0,851 \\
\hline \multicolumn{2}{|l|}{ Bem-estar emocional $(\alpha=0,943 ; n=151)$} \\
\hline 18. Sentir-se deprimida & 0,874 \\
\hline 19. Sentir-se com vontade de chorar & 0,883 \\
\hline 20. Sentir-se extremamente infeliz & 0,803 \\
\hline 21. Alterações de humor & 0,874 \\
\hline 22. Sentir-se mal-humorada ou irritável & 0,872 \\
\hline 23. Sentir-se violenta ou agressiva & 0,667 \\
\hline \multicolumn{2}{|l|}{ Suporte social $(\alpha=0,912 ; n=152)$} \\
\hline 24. Incapacidade de dizer como se sente & 0,764 \\
\hline 25. Sentir que os outros não compreendem & 0,857 \\
\hline 26. Sentir que os outros pensam que se está a lamuriar & 0,854 \\
\hline 27. Sentir-se sozinha & 0,735 \\
\hline \multicolumn{2}{|l|}{ Autoimagem $(\alpha=0,876 ; n=152)$} \\
\hline 28. Não poder vestir as roupas que queria & 0,758 \\
\hline 29. Sentir aparência afetada & 0,810 \\
\hline 30. Falta de confiança & 0,717 \\
\hline
\end{tabular}

e a sua aplicação clínica para avaliação da qualidade de vida das mulheres portuguesas com endometriose.

A amostra de doentes em que foi aplicado o questionário apresentava idade média (34,7 anos) que está de acordo com a idade média das mulheres portuguesas na faixa etária dos 18 aos 49 anos de idade - 34,65 anos. ${ }^{31}$ Relativamente às habilitações literárias, $40,8 \%$ concluíram o ensino secundário e $38,2 \%$ tinham habilitações iguais ou superiores a uma licenciatura, o que representa uma amostra com elevado nível de escolaridade para Portugal.
De facto, de acordo com os Censos 2011, a população portuguesa com 23 ou mais anos, que possui ensino superior completo representa cerca de $15 \%$, dos quais $60 \%$ são muIheres. ${ }^{32}$ Contudo, o nível de habilitações literárias é inferior ao descrito para outras populações, como por exemplo a brasileira. Bellelis et al, num estudo retrospectivo sobre aspetos epidemiológicos e clínicos da endometriose pélvica, incluindo 892 doentes, descrevem que 51,9\% das mulheres possuíam o terceiro grau de ensino completo (correspondente ao ensino superior). ${ }^{33}$ 
De acordo com a classificação da ASMR, todos os estádios de endometriose se encontram representados na amostra do presente trabalho. Contudo, a maioria das mulheres apresenta endometriose no estádio IV. Tal deve-se ao facto de a consulta em que os questionários foram aplicados ser uma consulta de Ginecologia Cirúrgica referência no país para doentes com endometriose, sobretudo com situações avançadas da doença. $\mathrm{Na}$ endometriose é amplamente reconhecida a ausência de correlação entre a intensidade dos sintomas (e consequentemente piores índices de qualidade de vida) e a gravidade da doença, ${ }^{1}$ traduzida neste trabalho pela classificação cirúrgica da ASMR. Assim, não existe evidência que a distribuição assimétrica da amostra, no que diz respeito a estádios cirúrgicos, influencie a avaliação da qualidade de vida realizada.

No âmbito da análise fatorial exploratória, efetuada com o objetivo de verificar as dimensões produzidas pela análise realizada no desenvolvimento original do questionário, foi possível verificar a estrutura do questionário central em cinco dimensões. Porém seis itens foram saturados em outro fator para além do fator hipotetizado, ou seja para além da sua dimensão original. De facto, os itens da dimensão controlo e impotência 'em geral, sentiu-se mal', 'sintomas não estão a melhorar', 'incapacidade de controlar sintomas', 'sintomas a controlar a vida' e 'sintomas a tirar a vida' associaram-se também à dimensão dor. Tal foi, também, já descrito na análise fatorial secundária realizada em 2006 para a versão original ${ }^{34} \mathrm{e}$ não é surpreendente dado que a dor é um sintoma cardinal da endometriose podendo condicionar negativamente a percepção da doença. ${ }^{1}$ Para além disso, o item 'Incapacidade de dizer como se sente' associou-se à dimensão bem-estar emocional para além da dimensão suporte social. Esta associação foi, também, descrita na versão americana ${ }^{21}$ e é facilmente explicada dado que a capacidade de comunicar é dependente do bem-estar emocional.

De referir que a nova análise fatorial realizada confirmou que emergia uma componente única de segunda

Tabela 4 - Avaliação da consistência interna do questionário modular do EHP-30 ( $\alpha$ - Cronbach).

\begin{tabular}{|c|c|}
\hline Dimensões & Correlação item-total \\
\hline \multicolumn{2}{|l|}{ Vida no trabalho $(\alpha=0,951 ; n=117$ em 120) } \\
\hline 1. Ausência do trabalho & 0,843 \\
\hline 2. Incapacidade para cumprir deveres no trabalho & 0,924 \\
\hline 3. Embaraçada com sintomas no trabalho & 0,784 \\
\hline 4. Sentimento de culpa por ausência do trabalho & 0,891 \\
\hline 5. Preocupação por não ser capaz de fazer o trabalho & 0,896 \\
\hline \multicolumn{2}{|l|}{ Relação com os filhos ( $\alpha=0,916 ; n=54$ em 58) } \\
\hline 1. Dificuldade em cuidar dos filhos & 0,846 \\
\hline 2. Incapacidade de brincar com os filhos & 0,846 \\
\hline \multicolumn{2}{|l|}{ Relações sexuais $(\alpha=0,941 ; n=112$ em 115) } \\
\hline 1. Dores na relação sexual & 0,757 \\
\hline 2. Preocupação com a relação sexual & 0,910 \\
\hline 3. Evitar relação sexual & 0,860 \\
\hline 4. Sentir-se culpada por não querer ter relação sexual & 0,871 \\
\hline 5. Angustiada por não conseguir ter prazer na relação sexual & 0,813 \\
\hline \multicolumn{2}{|l|}{ Relação com os médicos ( $\alpha=0,948 ; n=121$ em 123) } \\
\hline 1. Médico não está a fazer nada por si & 0,937 \\
\hline 2. Médico acha que é tudo da sua cabeça & 0,897 \\
\hline 3. Angustiada com a falta de conhecimentos do médico & 0,872 \\
\hline 4. Sentir que faz perder tempo ao médico & 0,812 \\
\hline \multicolumn{2}{|l|}{ Tratamento $(\alpha=0,863 ; n=100$ em 103) } \\
\hline 1. Angustiada porque o tratamento não está a funcionar & 0,731 \\
\hline 2. Dificuldade em lidar com os efeitos secundários & 0,723 \\
\hline 3. Aborrecida com a quantidade de tratamentos & 0,771 \\
\hline \multicolumn{2}{|l|}{ Infertilidade $(\alpha=0,880 ; n=70$ em 71) } \\
\hline 1. Preocupada com a possibilidade de não ter (mais) filhos & 0,791 \\
\hline 2. Sentir-se diferente por não poder/ não conseguir ter (mais) filhos & 0,824 \\
\hline 3. Sentir-se deprimida com a possibilidade de não ter (mais) filhos & 0,793 \\
\hline 4. Sentir que a possibilidade de infertilidade provoca tensão na relação com o parceiro & 0,573 \\
\hline
\end{tabular}


ordem, que explica $78,0 \%$ de variância, à semelhança do verificado para a versão original, americana e iraniana. ${ }^{2,21,24}$ Tal traduz que as dimensões podem ser somadas para criar uma pontuação única.

A consistência interna do questionário foi elevada para todas as dimensões, quer do questionário central quer modular, apresentando um alfa de Cronbach superior a 0,7. $\mathrm{Na}$ verdade, oito das 11 dimensões $(72,7 \%)$ do EHP-30 apresentaram valores superiores a 0,9 , indicando que estas dimensões seriam adequadas para uma análise individual de doentes. Os valores de alfa de Cronbach apresentados neste estudo foram similares e mesmo superiores aos descritos no estudo original, com exceção das dimensões relação com os filhos $(0,916$ vs. 0,97$)$, relações sexuais $(0,941$ vs. 0,96$)$ e infertilidade $(0,880$ vs. 0,92$) .{ }^{2}$ Para além disso, foram superiores aos resultados apresentados pela versão de português do brasil em todas as dimensões. ${ }^{8}$

Ainda relativamente à comparação da versão do EHP30 aqui estudada com a versão de português do brasil, encontram-se diferenças essencialmente quanto à linguagem utilizada. De facto, em ambas as versões se procurou realizar uma tradução que assegurasse a equivalência semântica com o instrumento original, mas garantindo que se mantinha a relevância clínica e o sentido dentro da cultura portuguesa ou brasileira. Desta forma, dadas as diferenças linguísticas, a construção das perguntas foi frequentemente diferente. Por exemplo: para a questão da versão original 'Felt your symptoms are taking away your life?', ${ }^{2}$ na versão de português do Brasil a questão é 'Sentiu como se seus sintomas estivessem prejudicando a sua vida?'8 e na versão aqui estudada é 'Sentiu que os seus sintomas the estão a tirar vida?'. No que diz respeito às propriedades psicométricas avaliadas, as versões apresentam-se com coeficientes que traduzem excelente consistência interna e validade convergente, embora os testes utilizados tenham sido diferentes (SF-36v2 no presente estudo vs. WHOQOL-Bref e o Inventário de Depressão de Beck na versão de português do brasil). ${ }^{8}$

À semelhança do descrito em várias versões do EHP30 já validadas, ${ }^{16,17,21,24}$ a correlação item-total excedeu 0 coeficiente mínimo aceitável de 0,4 em todos os itens do questionário central e modular, o que representa uma boa consistência item-total.

No que diz respeito à avaliação da validade convergente, verificou-se uma associação negativa significativa entre

Tabela 5 - Correlação entre as dimensões dos questionários central e modular do EHP-30 e as dimensões do SF-36v2.

\begin{tabular}{llcc}
\hline EHP-30 & SF-36v2 & Correlação de Pearson & \multicolumn{1}{c}{$\boldsymbol{p}$} \\
\hline Dor & Dor corporal & $-0,739$ & $<0,001$ \\
Dor & Desempenho físico & $-0,556$ & $<0,001$ \\
Controlo e impotência & Funcionamento social & $-0,655$ & $<0,001$ \\
Controlo e impotência & Desempenho físico & $-0,559$ & $<0,001$ \\
Bem-estar emocional & Desempenho emocional & $-0,600$ & $<0,001$ \\
Bem-estar emocional & Saúde mental & $-0,738$ & $<0,001$ \\
Bem-estar emocional & Saúde geral & $-0,567$ & $<0,001$ \\
Suporte social & Funcionamento social & $-0,628$ & $<0,001$ \\
Vida no trabalho & Desempenho físico & $-0,627$ & $<0,001$ \\
\hline EHP-30: Endometriosis Health Profile-30; SF-36v2: MOS Short Form Health Survey - 36 Item - version 2; a: Correlações negativas pois os scores do EHP-30 e do SF-36v2 são em \\
direções diferentes.
\end{tabular}

Tabela 6 - Estatística descritiva das 5 dimensões do questionário central e 6 dimensões do questionário modular do EHP-30

\begin{tabular}{lcccccc}
\hline Dimensão & $\mathbf{n}$ & Média \pm DP & Mínimo & Máximo & Assimetria & Curtose \\
\hline Questionário Central & & & & & & \\
Dor & 147 & $37,11 \pm 30,32$ & 0 & 93,18 & 0,092 & $-1,503$ \\
Controlo e impotência & 150 & $40,94 \pm 30,43$ & 0 & 95,83 & 0,021 & $-1,373$ \\
Bem-estar emocional & 151 & $41,20 \pm 27,43$ & 0 & 95,83 & $-0,015$ & $-1,134$ \\
Suporte social & 152 & $40,21 \pm 29,17$ & 0 & 100,00 & 0,004 & $-1,200$ \\
Autoimagem & 152 & $34,10 \pm 27,57$ & 0 & 100,00 & 0,303 & $-1,087$ \\
Questionário Modular & & & & 100,00 & 0,558 & $-1,122$ \\
Vida no trabalho & 117 & $28,89 \pm 30,09$ & 0 & 87,50 & 0,529 & $-1,148$ \\
Relação com os filhos & 54 & $27,78 \pm 29,00$ & 0 & 100,00 & $-0,109$ & $-1,171$ \\
Relações sexuais & 112 & $46,12 \pm 31,07$ & 0 & 100,00 & 2,079 & 3,325 \\
Relação com os médicos & 121 & $12,76 \pm 24,44$ & 0 & 100,00 & 0,418 & $-1,038$ \\
Tratamento & 100 & $30,92 \pm 28,46$ & 0 & 100,00 & $-0,394$ & $-0,617$ \\
Infertilidade & 70 & $55,89 \pm 28,92$ & 0 & & &
\end{tabular}


as dimensões similares do EHP-30 e do SF-36v2 (versão portuguesa previamente validada como instrumento de avaliação da qualidade de vida). Na verdade, o coeficiente de Pearson foi igual ou superior no presente estudo para todas as dimensões avaliadas, comparativamente com o estudo original. $^{2}$ Por exemplo, a correlação foi máxima em ambos os estudos entre a dimensão dor do EHP-30 e a dimensão dor corporal do SF-36v2, tendo sido de 0,73 em ambos. Também na versão chinesa do EHP-30, a correlação foi máxima entre estas dimensões e foi significativa nas sete correlações hipotetizadas. ${ }^{16}$ Por sua vez, na versão iraniana a correlação foi máxima entre a dimensão bem-estar emocional do EHP-30 e o desempenho emocional do $\mathrm{SF}-36 .^{24}$

A taxa de preenchimento do questionário, quer central quer modular, foi elevada, o que indica a aceitabilidade e fácil compreensão do mesmo. De facto, mesmo a dimensão relações sexuais, que poderia dado o seu caráter mais íntimo levantar mais reservas, apresentou uma taxa de preenchimento de 97,4\%. Já a versão do EHP-30 de português do brasil tinha demonstrado ser um instrumento de fácil e rápida aplicação, e bem aceite pelas doentes. ${ }^{8}$

No questionário central, à semelhança do descrito na versão iraniana do EHP-30, a dimensão bem-estar emocional apresentou a maior pontuação mediana e portanto o impacto mais negativo sobre a qualidade de vida. ${ }^{24}$ Por sua vez, tal como as versões americana e australiana, a dimensão autoimagem apresentou a menor pontuação mediana. ${ }^{21,22}$ No questionário modular, à semelhança das versões original, australiana, holandesa, iraniana e chinesa, a dimensão infertilidade apresentou a maior pontuação mediana e, consequentemente o impacto mais negativo sobre a qualidade de vida. 2,16,17,22,24 Portanto, de acordo com os nossos resultados e à semelhança da literatura, a redução do bem-estar emocional e a preocupação com uma possível infertilidade são os aspetos que mais condicionam a qualidade de vida da mulher com endometriose. Na literatura internacional, o impacto da endometriose na qualidade de vida das mulheres tem sido amplamente demonstrado e outros aspetos têm sido discutidos. . $^{1,5,10,11,35,36}$ Nnoaham et al descreveram num estudo, incluindo 1418 mulheres de 10 países diferentes, que a endometriose prejudica a qualidade de vida e a produtividade laboral, independentemente do país ou da étnia. ${ }^{10}$ Tais resultados foram corroborados por Fourquet et al ao demonstrarem que os sintomas relacionados com a endometriose condicionam absentismo e diminuição da produtividade laboral, bem como diminuição do bem-estar físico e mental das doentes. ${ }^{35} \mathrm{Um}$ estudo multicêntrico mais recente, incluindo 931 mulheres de 10 países, relatou um significativo efeito da endometriose no bem-estar físico, mental e social das doentes, nomeadamente no trabalho ( $51 \%$ das mulheres) e nas relações (50\% das mulheres). ${ }^{36}$ De referir que o EHP-30 permite avaliar estas diferentes dimensões da qualidade de vida, nomeadamente nas dimensões dor, controlo e impotência, bem-estar emocional, suporte social e vida no trabalho.

Relativamente a limitações do presente trabalho, devemos referir o facto de a fiabilidade teste-reteste e a sensibilidade à mudança do questionário não terem sido avaliadas. Apesar de coeficientes de correlação intra-classe aceitáveis terem sido descritos para diferentes versões, ,2,8,19,20 incluindo a versão original, em estudos futuros, a sensibilidade à mudança da versão portuguesa do questionário deverá ser analisada. Só desta forma poderemos concluir a capacidade da versão portuguesa do EHP-30 para medir o efeito de determinado tratamento na qualidade de vida das mulheres portuguesas com endometriose.

\section{CONCLUSÕES}

O presente estudo demonstra que a versão portuguesa do EHP-30 é um instrumento adequado, validado e bem aceite para a avaliação da qualidade de vida das mulheres portuguesas com endometriose. Esta versão permitirá avaliar objetivamente a qualidade de vida das mulheres portuguesas com endometriose, bem como a sua comparação com estudos internacionais em que o mesmo instrumento seja utilizado.

São necessários estudos adicionais para avaliar a fiabilidade teste-reteste e a sensibilidade à mudança desta versão portuguesa do EHP-30. A confirmar-se a sua adequação, este instrumento poderá posteriormente ser usado em estudos de avaliação da eficácia terapêutica.

\section{AGRADECIMENTOS}

Os autores agradecem a Pedro Lopes Ferreira pela autorização dada para utilização da versão portuguesa do SF-36v2. Tal permitiu realizar a avaliação da validade convergente da versão portuguesa do EHP-30.

\section{PROTECÇÃO DE PESSOAS E ANIMAIS}

Os autores declaram que o estudo foi aprovado pela Comissão de Ética do Centro Hospitalar Lisboa Norte Hospital Universitário de Santa Maria e se desenvolveu no respeito pela Declaração de Helsínquia da Associação Médica Mundial.

\section{CONFIDENCIALIDADE DOS DADOS}

Os autores declaram ter seguido os protocolos do seu centro de trabalho acerca da publicação dos dados de doentes.

\section{CONFLITO DE INTERESSES}

Os autores declaram não ter nenhum conflito de interesses relativamente ao presente artigo.

\section{FONTES DE FINANCIAMENTO}

Não existiram fontes externas de financiamento para a realização deste artigo. 
Giudice LC. Clinical practice. Endometriosis. N Engl J Med. 2010;362:2389-98

2. Jones G, Kennedy S, Barnard A, Wong J, Jenkinson C. Development of an endometriosis quality-of-life instrument: The Endometriosis Health Profile-30. Obstet Gynecol. 2001;98:258-64

3. Colwell HH, Mathias SD, Pasta DJ, Henning JM, Steege JF. A health-related quality-of-life instrument for symptomatic patients with endometriosis: a validation study. Am J Obstet Gynecol. 1998;179:4755.

4. Dubernard G, Rouzier R, David-Montefiore E, Bazot M, Darai E. Use of the SF-36 questionnaire to predict quality-of-life improvement after laparoscopic colorectal resection for endometriosis. Hum Reprod 2008;23:846-51.

5. Bodner $\mathrm{CH}$, Garratt AM, Ratcliffe J, Macdonald LM, Penney GC Measuring health-related quality of life outcomes in women with endometriosis--results of the Gynaecology Audit Project in Scotland. Health Bull. 1997:55:109-17.

6. Guyatt $\mathrm{GH}$, Feeny $\mathrm{DH}$, Patrick DL. Measuring health-related quality of life. Ann Intern Med. 1993;118:622-9.

7. Loverro G, Carriero C, Rossi AC, Putignano G, Nicolardi V, Selvaggi L. A randomized study comparing triptorelin or expectant management following conservative laparoscopic surgery for symptomatic stage III-IV endometriosis. Eur J Obstet Gynecol Reprod Biol. 2008;136:194-8.

8. Mengarda CV, Passos EP, Picon P, Costa AF, Picon PD. Validation of Brazilian Portuguese version of quality of life questionnaire for women with endometriosis (Endometriosis Health Profile QuestionnaireEHP-30). Rev Bras Ginecol Obstet. 2008;30:384-92.

9. FauconnierA, Chapron C. Endometriosis and pelvic pain: epidemiological evidence of the relationship and implications. Hum Reprod Update. 2005;11:595-606

10. Nnoaham KE, Hummelshoj L, Webster $P$, d'Hooghe $T$, de Cicco Nardone F, de Cicco Nardone C, et al. Impact of endometriosis on quality of life and work productivity: a multicenter study across ten countries. Fertil Steril. 2011;96:366-73.

11. Simoens S, Dunselman G, Dirksen C, Hummelshoj L, Bokor A, Brandes I, et al. The burden of endometriosis: costs and quality of life of women with endometriosis and treated in referral centres. Hum Reprod. 2012;27:1292-9.

12. Jones GL, Kennedy SH, Jenkinson C. Health-related quality of life measurement in women with common benign gynecologic conditions: a systematic review. Am J Obstet Gynecol. 2002;187:501-11.

13. Garry R, Clayton R, Hawe J. The effect of endometriosis and its radical laparoscopic excision on quality of life indicators. BJOG. 2000;107:4454

14. Miller JD. Quantification of endometriosis-associated pain and quality of life during the stimulatory phase of gonadotropin-releasing hormone agonist therapy: a double-blind, randomized, placebo-controlled trial. Am J Obstet Gynecol. 2000;182:1483-8.

15. Neelakantan D, Omojole F, Clark TJ, Gupta JK, Khan KS. Quality of life instruments in studies of chronic pelvic pain: a systematic review. J Obstet Gynaecol. 2004;24:851-8.

16. Jia SZ, Leng JH, Sun PR, Lang JH. Translation and psychometric evaluation of the simplified Chinese-version Endometriosis Health Profile-30. Hum Reprod. 2013;28:691-7.

17. van de Burgt TJ, Hendriks JC, Kluivers KB. Quality of life in endometriosis: evaluation of the Dutch-version Endometriosis Health Profile-30 (EHP30). Fertil Steril. 2011;95:1863-5.

18. Vincent K, Kennedy S, Stratton P. Pain scoring in endometriosis: entry criteria and outcome measures for clinical trials. Report from the Art and Science of Endometriosis meeting. Fertil Steril. 2010;93:62-7.

19. Jones $\mathrm{G}$, Jenkinson $\mathrm{C}$, Kennedy S. Evaluating the responsiveness of the Endometriosis Health Profile Questionnaire: the EHP-30. Qual Life Res. 2004;13:705-13

20. van de Burgt TJ, Kluivers KB, Hendriks JC. Responsiveness of the Dutch Endometriosis Health Profile-30 (EHP-30) questionnaire. Eur J Obstet Gynecol Reprod Biol. 2013;168:92-4.

21. Jenkinson C, Kennedy S, Jones G. Evaluation of the American version of the 30-item Endometriosis Health Profile (EHP-30). Qual Life Res. 2008;17:1147-52.

22. Khong SY, Lam A, Luscombe G. Is the 30-item Endometriosis Health Profile (EHP-30) suitable as a self-report health status instrument for clinical trials? Fertil Steril. 2010;94:1928-32.

23. Maiorana A, Scafidi Fonti GM, Audino P, Rosini R, Alio L, Oliveri AM, et al. The role of EHP-30 as specific instrument to assess the quality of life of Italian women with endometriosis. Minerva Ginecol. 2012;64:231-8.

24. Nojomi M, Bijari B, Akhbari R, Kashanian M. The assessment of reliability and validity of Persian version of the endometriosis health profile (EHP30). Iran J Med Sci. 2011;36:84-9.

25. D' Albergaria Martins C. Adaptação do Endometriosis Health Profile Questionnaire (EHP-30) para a cultura portuguesa. Dissertação de Mestrado (Fisioterapia em Saúde da Mulher). Alcoitão: Escola Superior de Saúde de Alcoitão; 2011.

26. Repositório de Instrumentos de Medição e Avaliação em Saúde (RIMAS); Centro de Estudos e Investigação em Saúde da Universidade de Coimbra (CEISUC). EHP-30. [Consultado 2013 Mar 01]. Disponível em: http://www.uc.pt/org/ceisuc/RIMAS/Lista/Instrumentos/EHP_30.

27. Hair JF Jr, Anderson RE, Tatham RL, Black WC. Multivariate data analysis. $5^{\text {th }}$ ed. New Jersey: Prentice Hall; 1998.

28. Ferreira PL, Santana P. Percepção de estado de saúde e de qualidade de vida da população activa: contributo para a definição de normas portuguesas. Rev Port Saúde Pública. 2003;21:15-30.

29. American Society for Reproductive Medicine. Revised American Society for Reproductive Medicine classification of endometriosis: 1996. Fertil Steril. 1997;67:817-21.

30. Wild D, Grove A, Martin M, Eremenco S, McElroy S, Verjee-Lorenz A, et al. Principles of Good Practice for the Translation and Cultura Adaptation Process for Patient-Reported Outcomes (PRO) Measures: report of the ISPOR Task Force for Translation and Cultural Adaptation. Value Health. 2005;8:94-104.

31. Instituto Nacional de Estatística. Inquérito à Fecundidade 2013. Lisboa; 2014. [Consultado 2014 Jul 5]. Disponível em http://www.ine.pt/xportal/ xmain?xpid=INE\&xpgid=ine_publicacoes\&PUBLICACOESpub_boui=2 18611955\&PUBLICACOESmodo $=2$

32. Instituto Nacional de Estatística. Censos 2011 Resultados Definitivos Portugal. Lisboa; 2012. [Consultado 2014 Jul 5]. Disponível em: censos. ine.pt/xportal/xmain?xpid=CENSOS\&xpgid=ine_censos_publicacao_ det\&contexto=pu\&PUBLICACOESpub boui=73212469\&PUBLICACO ESmodo=2\&selTab=tab1\&pcensos $=61969554$.

33. Bellelis P, Dias JA Jr, Podgaec S, Gonzales M, Baracat EC, Abrão MS Epidemiological and clinical aspects of pelvic endometriosis - a case series. Rev Assoc Med Bras. 2010;56:467-71.

34. Jones G, Jenkinson C, Taylor N, Mills A, Kennedy S. Measuring quality of life in women with endometriosis: tests of data quality, score reliability, response rate and scaling assumptions of the Endometriosis Health Profile Questionnaire. Hum Reprod. 2006;21:2686-93.

35. Fourquet J, Báez L, Figueroa M, Iriarte RI, Flores I. Quantification of the impact of endometriosis symptoms on health-related quality of life and work productivity. Fertil Steril. 2011;96:107-12.

36. De Graaff AA, D'Hooghe TM, Dunselman GA, Dirksen CD, Hummelsho L, WERF EndoCost Consortium, et al. The significant effect of endometriosis on physical, mental and social wellbeing: results from an international cross-sectional survey. Hum Reprod. 2013;28:2677-85. 
Cristina NOGUEIRA-SILVA, Patrício COSTA, Carla MARTINS, Sónia BARATA, Conceição ALHO, Carlos CALHAZ-JORGE, Filipa OSÓRIO

\section{Validação da Versão Portuguesa do Questionário EHP-30 (The Endometriosis Health Profile-30)}

Acta Med Port 2015:28:347-356

Publicado pela Acta Médica Portuguesa, a Revista Científica da Ordem dos Médicos

Av. Almirante Gago Coutinho, 151

1749-084 Lisboa, Portugal.

Tel: +351218428215

E-mail: submissao@actamedicaportuguesa.com

www.actamedicaportuguesa.com

ISSN:0870-399X | e-ISSN: 1646-0758

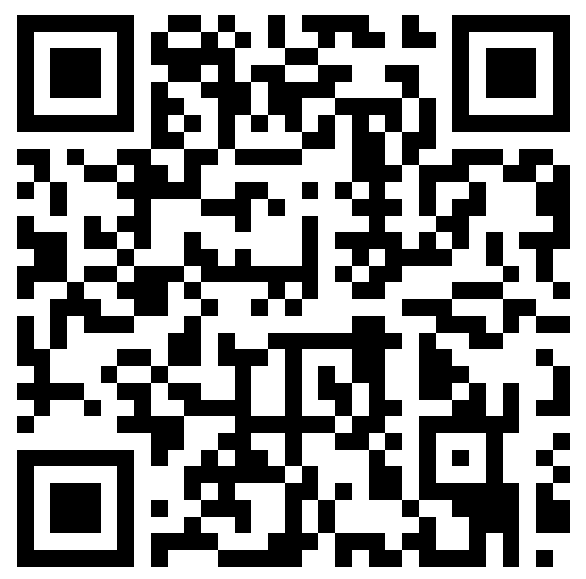

